\title{
A Signal Coordination Control Based on Traversing Empty between Mid-Block Street Crossing and Intersection
}

\author{
Changjiang Zheng, ${ }^{1}$ Genghua $\mathrm{Ma}^{2}{ }^{2}$ Jinhua $\mathrm{Wu}{ }^{1}$ \\ Xiaoli Zhang, ${ }^{1}$ and Xiaolei Zhang ${ }^{1}$ \\ ${ }^{1}$ College of Civil and Transportation Engineering, Hohai University, Nanjing 210098, China \\ ${ }^{2}$ College of Harbour, Coastal and Offshore Engineering, Hohai University, Nanjing 210098, China
}

Correspondence should be addressed to Jinhua Wu, wujinhua115@126.com

Received 29 June 2012; Revised 6 September 2012; Accepted 11 September 2012

Academic Editor: Wuhong Wang

Copyright (c) 2012 Changiiang Zheng et al. This is an open access article distributed under the Creative Commons Attribution License, which permits unrestricted use, distribution, and reproduction in any medium, provided the original work is properly cited.

To solve the problem in pedestrian Mid-Block street crossing, the method of signal coordination control between mid-block street crossing and intersection is researched in this paper. The paper proposes to use "distance-flow rate-time" graph as the tool for building coordination control system model which is for different situations of traffic control. Through alternating the linear optimization model, the system outputs the distribution of signal timing and system operational factors (delays in vehicles and mid-block street crossing). Finally, taking one section on the Taiping North Road in Nanjing as an example, the signal coordination control is carried out. And the results which are delays in the vehicles and mid-block street crossing are compared to those in the current distribution of signal timing.

\section{Introduction}

There are two kinds of representation methods in studying discrete feature of vehicles flow on the road, normal distribution function-Pacey method (1956) and geometric distribution function-Robertson method (1969) [1]. A. F. Rumsey and M. G. Hartley carried out computer simulations for comparison tests about these two distribution function methods proposed. They found although arrival rates of vehicle flow in downstream crossing which are calculated by those two different types of distribution functions are different, it is only a little effect on signal timing design. At present, the empirical formula that D. I. Robertson established based on the geometric distribution assumption is applied widely.

Studying pedestrian traffic characteristics, the American scholar JTFruit (1973) has researched minutely on pedestrian walking speed, density, and traffic flow, pedestrian spatial 
characteristics, and their relationships in this doctoral thesis, Pedestrian Planning and Design. He also proposed recommended value of the level of service of the sidewalk; Japanese scholars, Hosoi et al. have conducted a study on pedestrians behaviour in the crowd [2]. Wang et al. have carried out simulation and analysis of driver's deceleration and acceleration behaviour under different driving situations after identified the key safety-related parameters [3]. Wang et al. investigated driver's car-following safety behaviour in more detail and then developed an integrated safety approaching model and simulation procedures for devising traffic accident solution and evaluating urban traffic at the microscopic level [4]. Data of nearly 3,500 pedestrian crossing operations were collected at 27 crosswalks in the Greater Amman Area. Statistical analyses were conducted to reveal which factors significantly contribute to pedestrians' speeds [5].

Many foreign scholars have conducted many successful researches and applications on coordination control of traffic signal in urban major trunk roads. In early period, through theoretical analysis, scholars did research on coordination control of traffic signal using the time-distance diagram for timing-type liner control system [6].

With the application of computers, people could use a computer to get program of control which might be difficult to be achieved manually. "Off-line" and "on-line" are the two computer methods to be used to calculate coordinate liner control system. Off-line method is prepared for computing software which is in the light of some kind of optimized principles. Liner timing methods can be developed by computer. Then these control programs are set to the signal control machine which makes the signal lamps be in operation in accordance with the timing program set from time to time. With these methods, computing software becomes the point which scholars focus on. Among these softwares, MAXBAND and PASSER II are more representative [7]. In on-line method, the timing Programs of liner control system are obtained by computing software, and input data computing software needed is directly received from the vehicle detector through computer. Also the signal lights operate under computer. It adopts two kinds of methods which are "Type of Timing programs Selection" and "Type of Timing Programs Production." Coordination control system is represented by the SCOOT, SCAT, UTCS, and RT-TRANCS.

Sun et al. establish a model of motorist-pedestrian. The model uses a binary logit approach; multiattribute regression analyses are performed to capture the decision making process of the pedestrian [8]. Wolshon and Taylor analyze the differences in certain delay parameters which would occur as a result of implementing SCATS signal control [9]. Trabia et al. present the design and evaluation of a fuzzy logic traffic signal controller for an isolated intersection [10]. In adaptive method, the phase, and green signal time of single intersection were set by fuzzy controller. Then the average delay of vehicles was taken as objective function, the green time of every single intersection was dynamically adjusted, and the phase shift was optimized through genetic algorithm [11].

Domestic and foreign scholars have did some research and application on coordination control between pedestrian mid-block street crossing and intersections, but not conducting systemic and deep studies and not forming a complete research results. Foreign scholars studied them from the perspective of speed discreteness. Domestic researches were different from them. In the view of concentration, Canqi and Peikum (1996) proposed diffusion models of traffic platoon, diffusion models of traffic head, and diffusion models of traffic rear [12]. Domestic scholars have studied various aspects of pedestrian walking characteristics. They conduct some researches on pedestrians' walking speed, stride, and occupying space of walking, and so forth. 
In actual application, domestic liner control system usually uses foreign technology and methods. Beijing has conducted liner control test which had good effect. Then it was gradually applied in some typical road sections. Nanjing's urban traffic control system was completely and successfully developed by self-localization. It was fit for the compound traffic conditions of our country, low-density road network, and special city road condition with great disparity gap between two junctions. Equipping three kinds of modes that were realtime adaptive control, fixed timing, and noncable coordination control, the system could set seventy green-wave lines under exceptional circumstances.

In theoretical research, Sen-Fa et al. (1998), Southeast University, proposed hierarchical structure of urban traffic liner control system. On the first level, the cycle and green ratio of traffic signal of a single junction was determined by fuzzy logic controller. On the second level, the phase offset between two adjacent junctions was obtained by fuzzy phase controller. And between two levels there was a fuzzy switch to coordinate them. So as to achieve the purpose of reducing vehicle delay, the author presented a specific implementation method which could effectively reduce the queue length [13]. Yun-Tao et al., Tongji University (2003), proposed a design method which used to optimize phase offset of coordination control on urban main roads based on a genetic algorithm [14]. Chu et al. model the role of the street environment in how people cross roads in urban settings. Respondents were placed in real traffic conditions at the curbside of street blocks in the Tampa Bay area for a threeminute observation of the street environment [15]. Ye et al. develop a method to separate the impacts on pedestrian traffic among different factors. Focusing on an individual pedestrian traffic indicator-walking speed-three important human factors, that is, gender, age, and luggage-carrying, were selected to investigate their respective impacts on walking speed [16].

\section{Building the System Model}

Large numbers of pedestrians crossing streets have become a significant feature of China's urban roads as travel increases. To solve this problem, many pedestrian mid-block crossings are set between adjacent intersections for the convenience of pedestrians. While most of the domestic pedestrian mid-block crossings lack reasonable and effective control, arterial traffic subject to certain constraints and obstacles.

On a section between two intersections, if a pedestrian mid-block crossing is controlled by one or more fixed-time and actuated signal controllers, coordination control of signal timing between pedestrians and intersections can be taken into consideration, which could reduce delays of vehicles on pedestrian mid-block crossings and improve safety of crossing streets.

Building a traditional artery or network coordination control models needs a "distance-time" graph [6], which is an intuitive and effective tool for coordination control studies. However, the author considers that it is not enough to use a "distance-time" graph. Traffic flow movement graph, also known as "flow rate-time" graph of traffic flow on sections, reflects the signal coordination relationship of pedestrian mid-block crossings and intersections as well. This paper will build a signal coordination control system model of pedestrian mid-block street crossings and intersections under different traffic control circumstances and take four pedestrian mid-block street crossings as an example which are on sections of the Taiping North Road in Nanjing for proving the feasibility of this model. 


\subsection{Building the "Distance-Time-Flow Rate" Graph}

Not only the "distance-time" graph but also the traffic flow movement graph which is "flow rate-time" graph of traffic flow on sections reflect the signal coordination relationship of pedestrian mid-block crossings and intersections. The changes of the traffic flow can be observed directly from the flow graph.

The "vehicle arrival rate-time" relational graph of the stop line sections is a basis to determine all the traffic flow movement parameters (queue length, delay time, stop rate). For this reason, the traffic model which the signal coordination between the pedestrian midblock street crossing and intersection relies on must be established on the basis of prediction of "vehicle arrival rate-time" graph.

If we added "flow rate-time" graph which reflects the flow rate changing to "distancetime" graph, and building the "distance-time-flow rate" graph of traffic flow movements, this would help a lot in finding the reasonable vehicle passing bandwidth and coordinating the initiate program. Besides, it also reflects system process parameters such as stop rate directly.

The steps to build a "distance-time-flow rate" graph (see Figure 1) between two sections are as follows.

(1) Define principal vertical axis as distance (line out the relative positions of stop lines on sections), auxiliary vertical axis as flow rate, and horizontal axis as time, and build a graphic formula.

(2) Draw a traffic flow rate-time graph of an initial traffic flow which comes from upstream sections.

(3) Take a predicted drive-in vehicle flow rate-time graph on downstream sections on the basis of certain functional relations.

(4) Design the distribution program of signal timing.

(5) Set the starting point of pedestrian cycle signal on mid-block street crossings according to the designed green time offset.

(6) Draw the signal phase diagram of pedestrian mid-block street crossings according to the signal timing distribution design and green time offset vehicle.

From Figure 1, all the traffic flows that arrive on the stop line are determined by a discharging law of upstream intersections. This means that if the functional relation between vehicle drive-out flow rate and time is known, we can calculate the functional relation between vehicle arrival rate and time on the stop lines. Speed discreteness should be considered when calculating the vehicle arrival flow rate-time graph on the stop line of pedestrian mid-block crossings, which is on the basis of drive-out rate graph of departing vehicles from upstream intersections. This paper uses geometric distribution functionRobertson [2] method to calculate vehicle arrival rate in a time interval:

$$
q_{\partial}(i+t)=F q_{0}(i)+(1-F) q_{\partial}(i+t-1)
$$

(see [17]). Revise (2.1),

$$
q_{\partial}(j)=\sum_{i=1}^{j-t} q_{0}(i) F(1-F)^{j-t-i}
$$




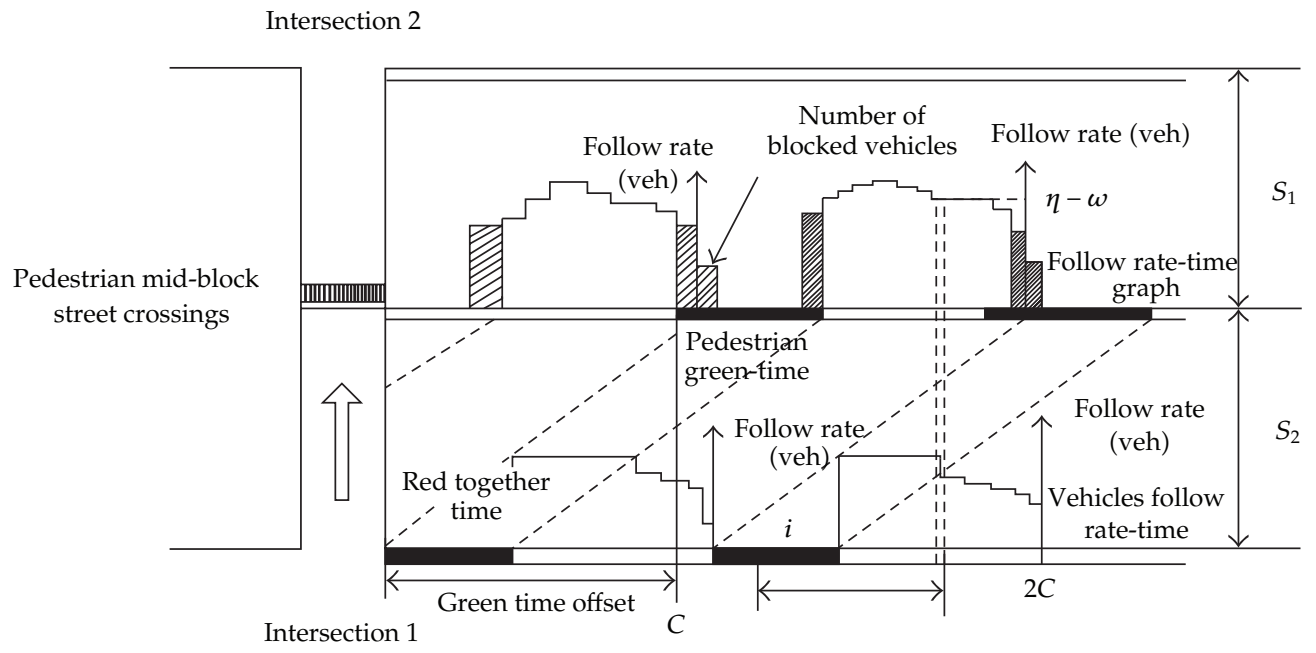

Figure 1: Distance-time-flow rate graph between two sections.

where $j=t+i . q_{\partial}(i+t)$-predicted vehicle arrival rate on a downstream section at $(i+t)$ time interval; $q_{0}(i)$-vehicle passing rate on the upstream stop line section at $i$ time interval; $t-0.8$ times of vehicle average travel time which is between two sections above, using the quantity of time intervals as a unit; $F-$ a factor which describes the extent of speed dispersion of traffic flow in the movement process, which is called traffic flow coefficient of dispersion, given by an empirical formula [18] as follows:

$$
F=\frac{1}{1+0.35 t}
$$

For a more convenient calculation, we divide a signal cycle into several smaller time periods. In these small time periods, it is supposed that the flow rate-time relation of vehicles arriving at intersections is of uniform distribution, which means to maintain the same value of flow rate which could be obtained by investigation. We can get a flow rate-time graph according to an average flow rate in a smaller period of time. And this could determine the vehicle flow rate which is from upstream intersections into road section of entry at the $i$ time interval.

\subsection{Calculate the Coordination Control of Pedestrian Mid-Block Street Crossing}

To define the signal timing program of pedestrian mid-block street crossing needs to determine several controlling parameters, which are signal cycle length and green time of pedestrian mid-block crossings and phase offsets.

The cycle length and signal stages of pedestrian mid-block street crossings could be determined by "flow-rate-time" graph and constraint conditions of limit value of waiting time which pedestrian could tolerate. And these things above are confirmed by the predicted traffic flow that arrives at pedestrian mid-block street crossings. 
For arterial roads and secondary arterial roads studied here, the road section traffic flow is of high volume and the vehicle speed is fast. As a result, the waiting time that pedestrian can tolerate is $50-60$ seconds.

The flow rate of vehicles that enter the intersection is related to the phase design of an intersection signal cycle, so the predicted flow rate-time graph that arrives at the pedestrian mid-block street crossings is related with the phase of the upstream signal cycle. There are three trickles of vehicles entering the intersection from upstream road section, which are straight flow $(S)$, right-entering right-turn flow $(R)$, and left-entering left-turn flow $(L)$. After merged, they are divided into five groups. Group 1 is $(R S L)$ that 3 trickles of traffic flow are in the same phase, group 2 is $(R / S / L)$ that 3 trickles of traffic flow are in different phases, group 3 is $(R S / L)$ that $R$ and $S$ are in the same phase, group 4 is $(S L / R)$ that $S$ and $L$ are in the same phase, and group 5 is $(R L / S)$ that $R$ and $L$ are in the same phase.

When it is RSL, usually the signal cycle length of an intersection is short. As a result, the intersection cycle could be the pedestrian signal cycle $C_{P}$. So, there is a "unimodal" in the vehicle traffic flow rate in a cycle, which concludes that the green time of pedestrian midblock street crossings $g_{p}$ can be set by using the gap that is formed by "red together time" of the upstream signal. See Figure 2.

Under such circumstance, the pedestrian signal is the intersection signal cycle, and the green time of pedestrian mid-block street crossing is a function of cycle length. Hence, determining phase offset is necessary for the pedestrian signal distribution. We build a unary linear programming model to solve the optimal phase offset which is in (2.4). The planning intention is to make total delay of vehicles $D_{r}$ in pedestrian green time minimum. The limit value of waiting time which pedestrians could tolerate is 60 seconds:

$$
\begin{gathered}
\min D_{r}=\sum_{j=\left(\mathrm{OFS}+C_{P}-g_{p}\right) / n}^{\left(\mathrm{OFS}+C_{P}\right) / n} n q_{j}\left(\mathrm{OFS}+C_{P}-n_{j}\right), \\
\bar{t}-\frac{C_{P}}{2} \leq \mathrm{OFS} \leq \frac{C_{P}}{2}+\bar{t} \\
g_{P}=f\left(C_{p}\right), \\
0 \leq C_{p}-g_{p} \leq 60, \\
g_{p}=\frac{Q_{p \max }}{e_{p r} \times d}+\frac{l}{v_{p}}=\frac{\left(a_{p}+\alpha a_{b}+\beta a_{h}\right) \times C_{p}}{e_{p r} \times d}+\frac{l}{v_{p}}=f\left(C_{p}\right),
\end{gathered}
$$

(see [6]), where $D_{r}$-delay of vehicles when it is green time for pedestrians and red time for vehicles (s); OFS - phase offset (s); $C_{P}$ - cycle length of pedestrians, equaling the cycle length of intersections $(\mathrm{s}) ; g_{p}$ - green time of pedestrians $(\mathrm{s}) ; j$-time interval in the green time; $q_{j}$-predicted flow rate of vehicles passing through stop lines of pedestrian mid-block street crossings which $j$ corresponds with; $n$-length of time intervals (s); $\bar{t}$-average travel time which is from upstream intersections into stop lines of pedestrian mid-block street crossings on road sections, calculated by the average travel speed of road sections (s); $Q_{p \max }$-number of traffic entities which is in a larger demand direction in a signal cycle, converted into units of persons (person); $a_{p}, a_{b}, a_{h}$-arrival rate of pedestrians, bicycles and help-move-vehicles separately in the large demanding direction (number of the arrivings/s); $\alpha, \beta$-separate coefficient of conversion of bicycles, help-move-vehicles, and pedestrians $(\alpha=5.6, \beta=5.6$ ); 


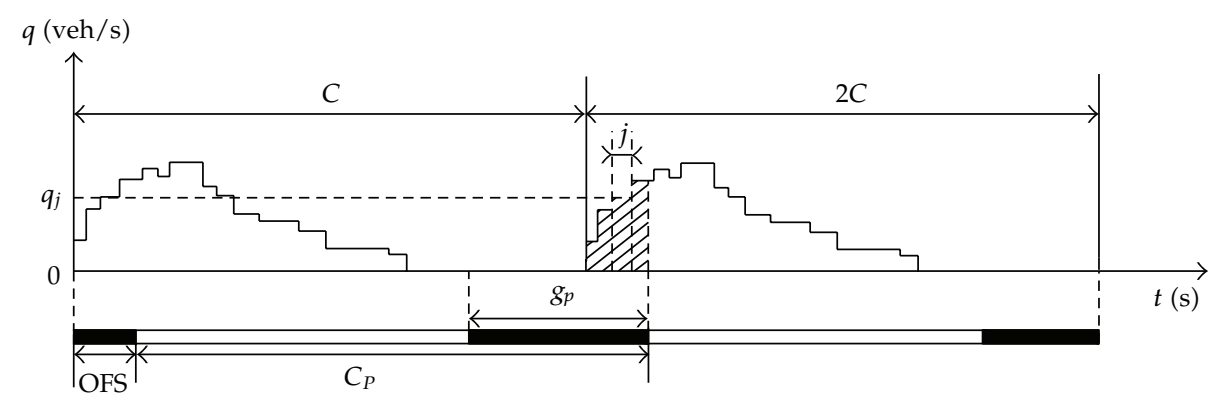

Figure 2: The establishment of first system optimal model on the basis of vehicle flow-time of crosswalk (signal single cycle of pedestrian).

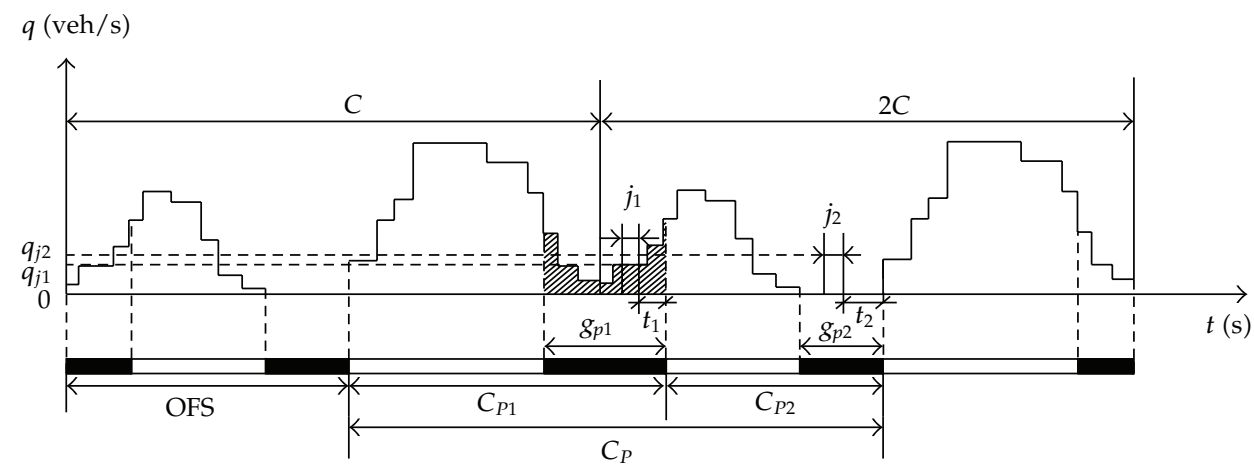

Figure 3: The establishment of first system optimal model on the basis of vehicle flow-time of crosswalk (signal double cycles of pedestrian).

$e_{p r}$-actual serving capacity of pedestrian mid-block crossings (person/green hour $\cdot \mathrm{m}$ ); $d$ width of pedestrian mid-block crossings $(\mathrm{m})$; l-length of pedestrian mid-block crossings, $(\mathrm{m}) ; v_{p}$-average speed of the pedestrian mid-block street crossing, taking it as 1.2 here $(\mathrm{m} / \mathrm{s})$; when it is $R / S / L$, the signal cycle is longer. There are "two humps" in a signal cycle. Besides, there is no gap that is formed by "red together time," see Figures 3 and 4 . In this circumstance, two departing opportunities can be given in one cycle for pedestrians. To set two green time of pedestrian mid-block street crossings $g_{1}, g_{2}$, we can use the peak valley between "two humps." This means to divide a cycle into two subcycles $C_{P 1}, C_{P 2}$, the sum of which equals the length of the intersection signal cycle. There is a pedestrian departing opportunity separately in each subcycle, and the length of each can be different.

On this occasion, the controls parameters are phase offset, length of subcycles and green time of the pedestrian signal. We could solve these parameters by solving a ternary linear programming model. Build the model like (2.7). This model is as a purpose of minimizing the vehicle delay $D_{r}$ in the green time of pedestrians, and the constraint conditions are that the length of the intersection signal cycle is the sum of subcycles, and the sum of two waiting time of pedestrians is no more than the limit value (here is $60 \mathrm{~s}$ ). 


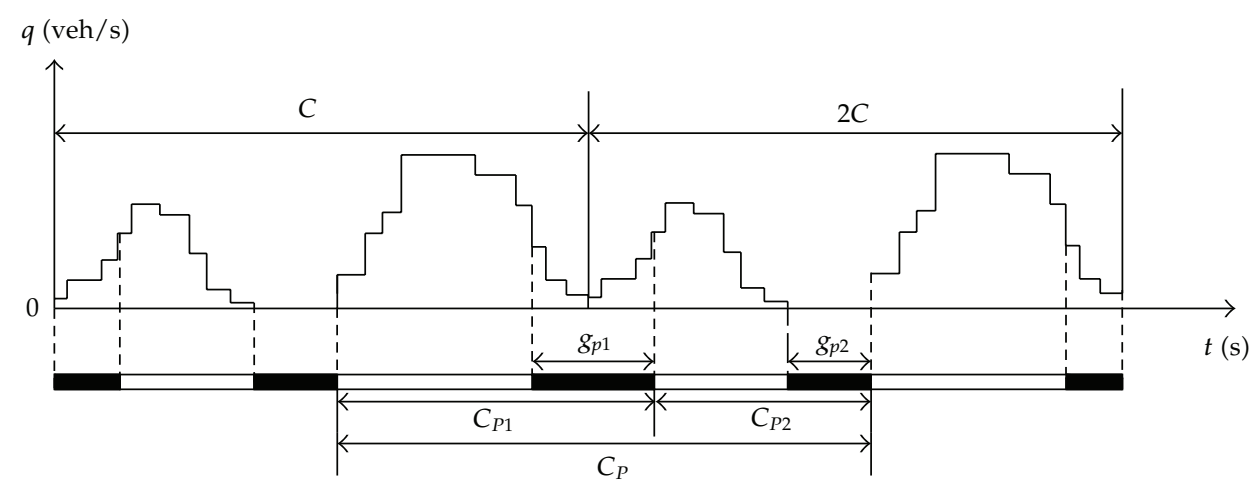

Figure 4: The establishment of first system optimal model on the basis of vehicle flow-time of crosswalk (signal double cycles of pedestrian).

There are three unknown variables and three constraint conditions:

$$
\begin{gathered}
\min D_{r}=\sum_{j_{1}=\left(\mathrm{OFS}+C_{P 1}-g_{p 1}\right) / n}^{\left(\mathrm{OFS}+C_{P 1}\right) / n} n q_{j_{1}} \times t_{1}+\sum_{j_{2}=\left(\mathrm{OFS}+C_{P}-g_{p 2}\right) / n}^{\left(\mathrm{OFS}+C_{P}\right) / n} n q_{j_{2}} \times t_{2}, \\
\mathrm{OFS}+C_{p 1}-n j_{1}=t_{1} \\
\mathrm{OFS}+C_{p}-n j_{2}=t_{2}, \\
C_{p 1}+C_{P 2}=C_{P} \\
g_{P 1}=f\left(C_{P 1}\right) \\
g_{P 2}=f\left(C_{P 2}\right) \\
0 \leq C_{P 1}-g_{P 1} \leq 60 \\
0 \leq C_{P 2}-g_{P 2} \leq 60 \\
\bar{t}-\frac{C_{p}}{2} \leq \mathrm{OFS} \leq \frac{C_{p}}{2}+\bar{t}
\end{gathered}
$$

where the meanings of $D_{r}$, OFS, $C_{P}, n, \bar{t}$ are the same; $C_{P 1}, C_{P 2}$ - subcycle length, dimidiate subcycle length $(\mathrm{s}) ; g_{p 1}, g_{p 2}$ - green time of pedestrians in subcycles and dimidiate subcycles (s), expressed by (2.6); $j_{1}, j_{2}$-time intervals in green time of subcycles and dimidiate subcycles; $q_{j_{1}}, q_{j_{2}}$-predicted traffic flow rate of stop lines through pedestrian mid-block street crossings which $j_{1}$ of $j_{2}$ matches along with; the situations of $R S / L, S L / R, R L / S$ are similar to $R / S / L$ situation. Therefore, we can solve the controls parameters in the same way.

An initial solution should be given when solving the linear programming model. By using an iteration procedure, we can calculate the length of subcycles of the pedestrian signal and the green ratio under minimum vehicle delay. And then the length of the green time is obtained in a subcycle. If the value of the initial solution is rational, which means 
the solution direction is correct, the computing time could be cut down. Usually the initial variable quantities are assigned as follows:

$$
\mathrm{OFS}=\frac{S_{1}}{v_{d}}, \quad C_{p 1}=C_{p 2}=\frac{1}{2} C_{p},
$$

where $S_{1}$-distance from road sections to stop lines of pedestrian mid-block crossings that comes from upstream intersections $(\mathrm{m}) ; v_{d}$-design speed on sections, taking the average speed value which has the highest frequency appearances; green flash time should also be set for warning the green time of pedestrians is going to finish. Usually it is $3-5$ seconds.

\section{Output of the System Model}

\subsection{Total Delay of Vehicles $D$}

In the objective function of the system, $D_{r}$ is the delay of vehicles when it is the pedestrian red light. But, in the beginning period of green time, the total delay of vehicles also contains $D_{s}$ which is caused by the subsequent arriving vehicles blocked in a discharging red time. $D_{s}$ is calculated by the delay time of vehicles [19] on the basis of the number of cumulative arriving of vehicles and the departing rate in green time. As a result, the total delay of vehicles is $D=D_{r}+D_{s}$.

If a cycle has two subcycles, calculating the total delays of subcycles, then the total vehicles' delays in a cycle are the sum of the total delays of subcycles.

\subsection{Average Delay of Vehicles}

The computing formula is

$$
\bar{D}=\frac{D}{Q}
$$

where $\bar{D}$ —average delay of vehicles (s/pcu); $D$ —total delay of vehicles (s); $Q$-number of vehicles passing the stop lines in a cycle (pcu).

\subsection{Stop Rate of Vehicles}

In a cycle of vehicles driving on a section, stop rate of vehicles is a ratio of the number of vehicles which stop on the stop lines of the pedestrian mid-block crossings and the number of all vehicles passing the pedestrian crossing lines. The stopping rate of vehicles on a pedestrian crossing is calculated as follows:

$$
E=\frac{\text { the number of vehicles stopping in a cycle }}{\text { the number of vehicles passing in a cycle }} \text {. }
$$




\subsection{Total Delay of Pedestrians}

Delay of a single person involves waiting time and the reaction time of crossing green light. When calculating the total delays of pedestrian, we only calculate the sum of waiting time of pedestrians as the total delay of pedestrians $D_{p}$. According to the formula that calculates the crossing green time, we take the product [20] of the actual service ability and the width of pedestrian mid-block crossings as the dissipation rate, which is $e_{p r} d / 3600$. The computing formula of unidirectional pedestrian crossing delay in a cycle is

$$
D_{P i}=\frac{e_{p r} d a_{i}\left(C_{P}-g_{p}\right)^{2}}{2\left(e_{p r} d-3600 a_{i}\right)},
$$

where $D_{P i}$-delay of unidirectional pedestrian mid-block crossings $(s)(i=1,2) ; a_{i}$-arrival rate of unidirectional pedestrians (person/s) $(i=1,2)$.

For a pedestrian crossing, the total delays of pedestrian mid-block crossings are the sum of $t$ bidirectional pedestrians, where $D_{P}=D_{P 1}+D_{P 2}$.

\subsection{Average Delay of Pedestrians}

After calculating the total delay of pedestrians, we know the quantity of arrival pedestrians in a cycle. The average delay of pedestrians can be calculated by using the following formula:

$$
\bar{D}_{p}=\frac{D_{P}}{Q_{P}}=\frac{D_{P 1}+D_{P 2}}{C_{P}\left(a_{1}+a_{2}\right)}
$$

$a_{i}$-arrival rate of unidirectional pedestrians, (person/s), $(i=1,2)$.

When there are several pedestrian mid-block crossings, the total delay of vehicles, the average delay of vehicles, the stop rate of vehicles, and the total delay of pedestrians all take the sum of delays of the pedestrian mid-block street crossings while the average delays of pedestrians are the geometry average value of all the average delays of pedestrian mid-block street crossings.

\section{Example Study}

\subsection{Investigation and Analysis of Taiping North Road}

\subsubsection{The Actuality of the Road Layout}

Taiping Road is a main road of Nanjing. The road layout diagram of Taiping Road (ZhujiangBeijing East Road) actuality is shown in Figure 5. This section has three T-shaped intersections which include an actuated pedestrian mid-block street crossing, a pedestrian overpass, and an uncontrolled pedestrian midblock street crossing. 


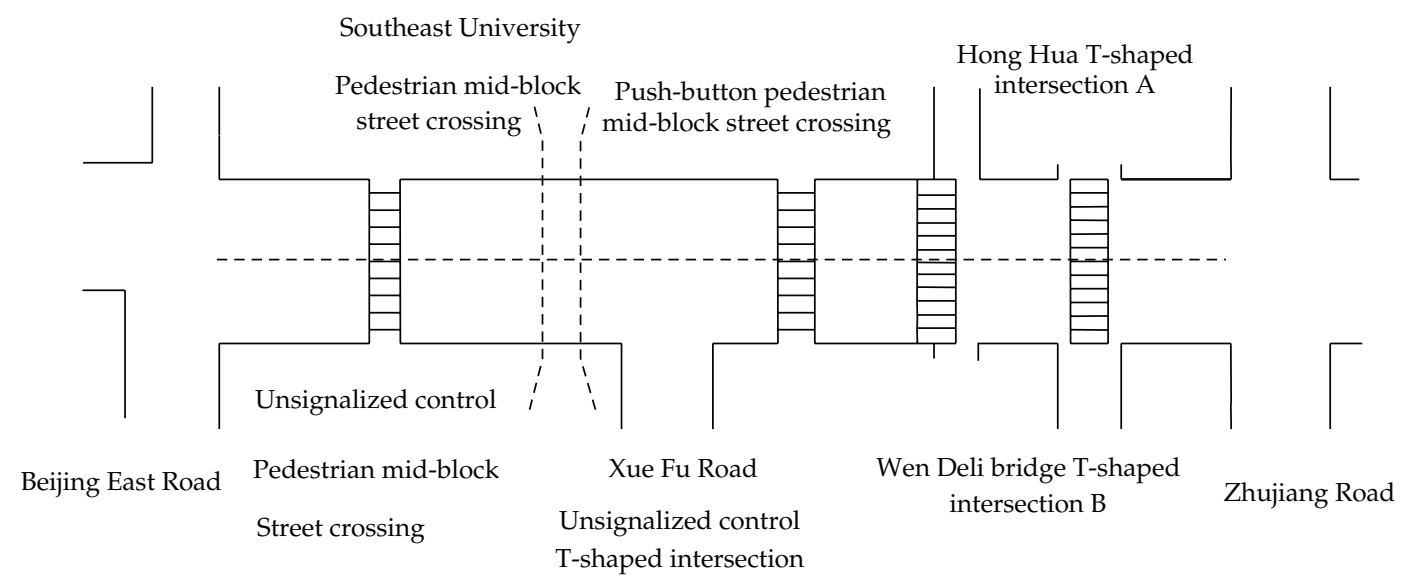

Figure 5: The road layout diagram of Taiping Road (Zhujiang-Beijing East Road) actuality.

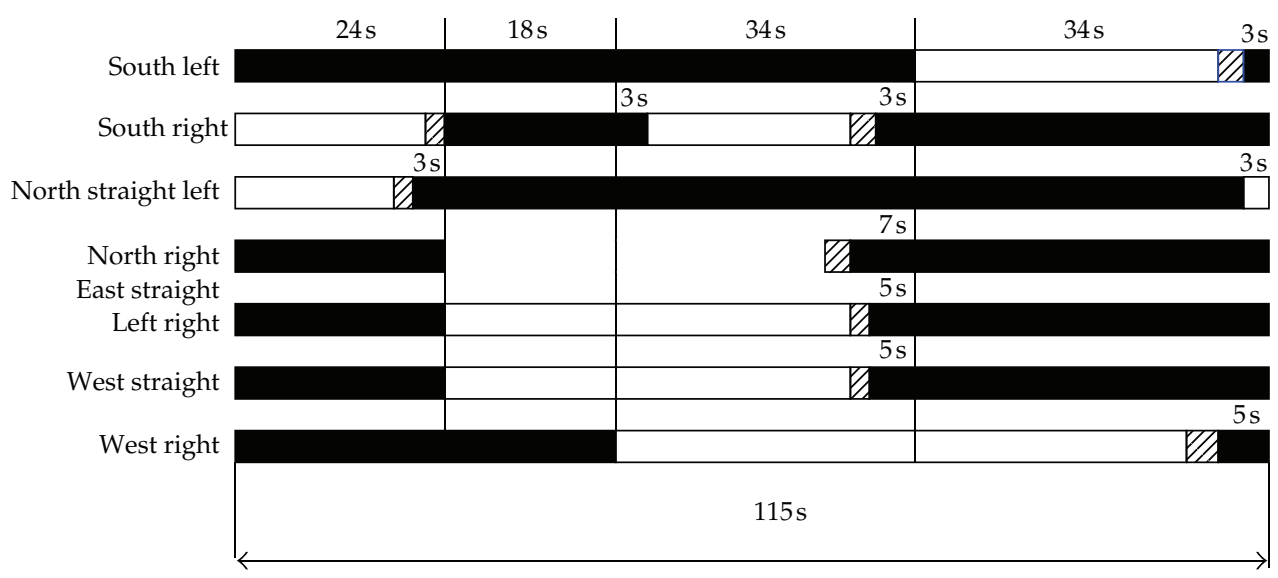

Figure 6: Distribution diagram of signal timing of Taiping North Road-Beijing East Road intersection.

\subsubsection{Distribution of Signal Timing and Road Information}

The timing signal controlled intersections on the section are Taiping North Road-Zhujiang Road intersection, Taiping North Road-Beijing East Road intersection, T-shaped intersection $\mathrm{A}$, and T-shaped intersection B. The distribution sketch maps of signal timing are shown in Figures 6, 7, 8, and 9.

\subsubsection{Traffic Characteristics of Vehicle}

\section{(1) Vehicle Speed of Road Section}

Through the investigation of vehicle speed of Taiping North Road section, the highest frequency of the speed value group is $42.5 \mathrm{~km} / \mathrm{h}$. 


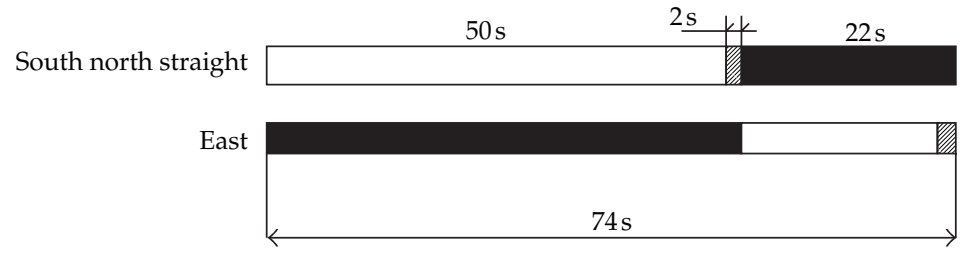

Figure 7: Distribution diagram of signal timing of T-shaped intersection A.

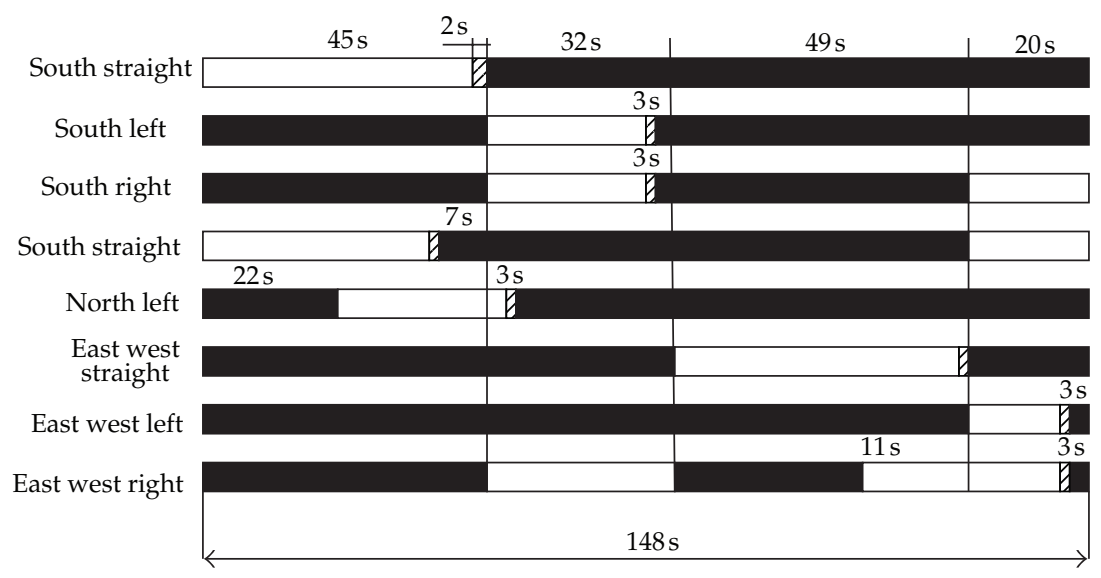

Figure 8: Distribution diagram of signal timing of Taiping North Road-Zhujiang Road intersection.

\section{(2) Vehicle Delay of Road Section}

Vehicle delay of road section is mainly produced in T-shaped intersection A and B and the actuated crosswalk $C$. The point investigation method is adopted to investigate vehicle delay at the peak time from the south to the north on the three intersections.

From the investigation and analysis of the three delays on Taiping Road section in the early peak time, the total delays of vehicles from south to north are $29673 \mathrm{~s}$, the average delay is $24.79 \mathrm{~s} / \mathrm{pcu}$, and the stop rates before the stop line separately are $0.375,0.34$, and 0.45 .

\subsubsection{Traffic Characteristics of Mid-Block Street Crossing}

\section{(1) Arrival Characteristics of Mid-Block Street Crossing}

Through surveys, the average traffic arrival rates of actuated crosswalk $\mathrm{C}$ and T-shaped junctions A and B are shown in Table 1.

\section{(2) Traffic Speed of Mid-Block Street Crossing}

Through investigation, the average mid-block street crossing speeds of pedestrians, bicycles and scooters are $1.2 \mathrm{~m} / \mathrm{s}, 2.33 \mathrm{~m} / \mathrm{s}$, and $2.1 \mathrm{~m} / \mathrm{s}$ on the road section. 


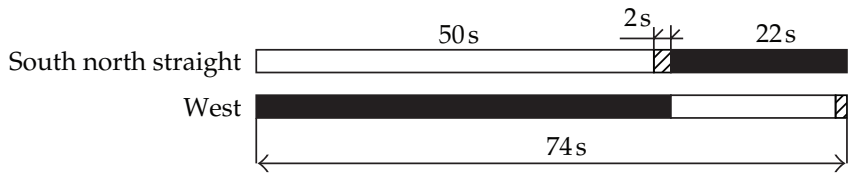

Figure 9: Distribution diagram of signal timing of T-shaped intersection B.

Table 1: The average traffic arrival rates of $\mathrm{C}, \mathrm{A}$, and B (person/s).

\begin{tabular}{lccc}
\hline Direction & & Crosswalk & \\
& Crosswalk C & T-shaped junction A & T-shaped junction B \\
\hline From east to west & 0.87 & 0.27 & 0.21 \\
From west to east & 0.12 & 0.18 & 0.20 \\
\hline
\end{tabular}

\section{(3) Traffic Delay of Mid-Block Street Crossing}

Pedestrian delays of T-shaped junctions A, B, and C at the early peak time are calculated in Table 2 .

\subsection{Unidirectional Signal Coordination Control between Pedestrian and Intersection in Taiping North Road}

A unidirectional signal coordination control between $B, A$, and C, three pedestrian mid-block street crossings, and the Taiping Road-Zhujiang Road intersection is realized on the road from south to north. First, B and upstream intersection are coordinated, then A and B are coordinated, finally $\mathrm{A}$ and the actuated crosswalk $\mathrm{C}$ are coordinated.

\subsubsection{Signal Coordination Control of Wen Deli Crosswalk B and the Pacific North Road-Zhujiang Road}

The signals of the upstream intersection into road section are merged in Figure 10.

Through investigation, the vehicle flow rate of the upstream intersection into road section is shown in calculation graph of section vehicle flow rate-time, taking $5 \mathrm{~s}$ as a time unit. The arrival flow rate-time graph of B stop line is acquired by (2.2).

The length of signal cycle of upstream intersection is $148 \mathrm{~s}$, and pedestrian signal adopts the same signal cycle of the upstream intersection. Because the signal cycle time is too long, the signal of the pedestrian mid-block street crossing adopts dual cycle. From the data in Table 2 and the relationship of entering flow rate and time on B stop line and through the controls parameter optimization model formula (2.4) and (2.6), we can obtain that

$$
\text { OFS is } 16 \mathrm{~s}, \quad C_{p 1} \text { is } 73 \mathrm{~s}, \quad C_{p 2} \text { is } 75 \mathrm{~s}, \quad \min D_{r} \text { is } 102 \mathrm{~s} / \text { cycle. }
$$

Then $g_{p 1}$ is $16 \mathrm{~s}$ and $g_{p 2}$ is $16 \mathrm{~s}$. The $5 \mathrm{~s}$ at the end of two green lights is designed into green flash time.

If saturation flow rate of cross-section is taken as $0.84 \mathrm{pcu} / \mathrm{s}$, then the total vehicle delay $D$ is $263 \mathrm{pcu} \cdot \mathrm{s} / \mathrm{cycle}$, that is $6397 \mathrm{pcu} \cdot \mathrm{s} / \mathrm{h}$. The average delay of vehicles at B is $\bar{D}=$ $D / Q=4.90$, and stopping rate is 0.17 . 
Table 2: Pedestrian actuality delays of B, A, and C.

\begin{tabular}{lcccccc}
\hline & \multicolumn{2}{c}{ B } & \multicolumn{2}{c}{ A } & \multicolumn{2}{c}{ C } \\
& $\begin{array}{l}\text { From east to } \\
\text { west }\end{array}$ & $\begin{array}{c}\text { From west } \\
\text { to east }\end{array}$ & $\begin{array}{c}\text { From east to } \\
\text { west }\end{array}$ & $\begin{array}{c}\text { From west } \\
\text { to east }\end{array}$ & $\begin{array}{c}\text { From east to } \\
\text { west }\end{array}$ & $\begin{array}{c}\text { From west } \\
\text { to east }\end{array}$ \\
\hline $\begin{array}{l}\text { Average delay } \\
\text { (s/person-period) }\end{array}$ & 24.5 & 24.4 & 25.3 & 24.5 & 20.4 & 33.6 \\
Total delay (s/period) & 416 & 395 & 553 & 357 & 1154 & 262 \\
\hline $\begin{array}{l}\text { The total delay per } \\
\text { hour of road section (s) }\end{array}$ & \multirow{2}{*}{35600} & & 40444 & 78425 \\
\hline
\end{tabular}

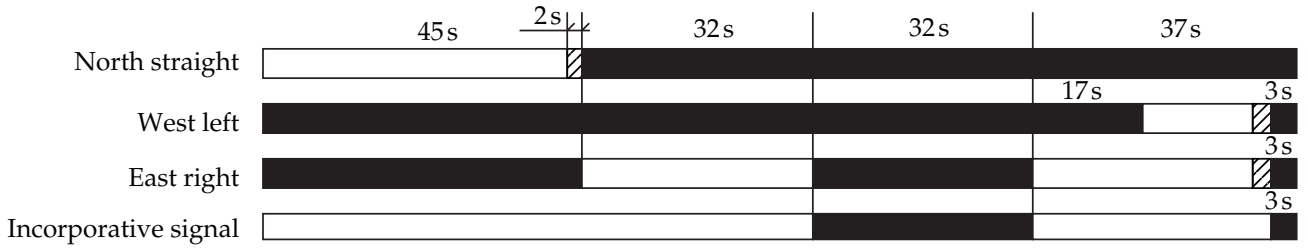

Figure 10: The merged signal of the upstream intersection into road section.

By (3.3) and (3.4), we can get that the average delay is $24.09 \mathrm{~s} /$ person from east to west, $25.2 \mathrm{~s} /$ person from west to east, and the total average delay is $24.6 \mathrm{~s} /$ person. Pedestrian total delay per hour of crosswalk B is $36413 \mathrm{~s}$.

\subsubsection{Signal Coordination Control between Hua Hongyuan Crosswalk A and Wen Deli Crosswalk B}

Through investigation, the exiting flow rates of B intersection are shown in calculation graph of section vehicle flow rate-time. Take $5 \mathrm{~s}$ as a time unit. The entering flow rate-time graph on A stop line is gained via (2.2). From the data in Table 3, the entrancing flow rate-time relation of A stop line, and the controls parameter optimization model formula (2.6) and (2.7) iteration, we can get that OFS is $21 \mathrm{~s}, C_{p 1}$ is $73 \mathrm{~s}, C_{p 2}$ is $75 \mathrm{~s}$, and $\min D_{r}$ is $8 \mathrm{~s} /$ cycle. Then $g_{p 1}$ is $16 \mathrm{~s}$ and $g_{p 1}$ is $16 \mathrm{~s}$. The $5 \mathrm{~s}$ at the end of two green lights is designed into green flash time.

If saturation flow rate is taken as $0.75 \mathrm{pcu} / \mathrm{s}$, then the total vehicle delay $\mathrm{D}$ is $277 \mathrm{pcu}$. $\mathrm{s} / \mathrm{cycle}$, that is $6757 \mathrm{pcu} \cdot \mathrm{s} / \mathrm{h}$. The average delay of vehicles at $\mathrm{A}$ is $\bar{D}=D / Q=5.17 \mathrm{~s} / \mathrm{pcu}$ and stop rate is 0.17 .

By (3.3) and (3.4), we can get that the average delay is $24.7 \mathrm{~s} /$ person from east to west, $24.0 \mathrm{~s} /$ person from west to east, and the total average delay is $24.3 \mathrm{~s} /$ person. Pedestrian total delay per hour of crosswalk A is $39624 \mathrm{~s}$.

\subsubsection{Signal Coordination Control Design of Southeast University Actuated Crosswalk C and Hong Huayuan Crosswalk A}

Through investigation, the exiting flow rates of A intersection are shown in calculation graph of section vehicle flow rate-time. Take $5 \mathrm{~s}$ as a time unit. The entering flow rate-time graph of C stop line is gained via (2.2). From the data in Table 3, the entering flow rate-time relation of 
Table 3: Traffic characteristic of B, A, and C intersections.

\begin{tabular}{lcccc}
\hline Crossing & & & Parameter & \\
& $l(\mathrm{~m})$ & $d(\mathrm{~m})$ & $v_{p}(\mathrm{~m} / \mathrm{s})$ & Average arrival rate (person/s) \\
\hline B & 18.6 & 6.5 & 1.2 & 0.21 \\
A & 18.6 & 5.6 & 1.2 & 0.27 \\
C & 18.6 & 5.6 & 1.2 & 0.87 \\
\hline
\end{tabular}

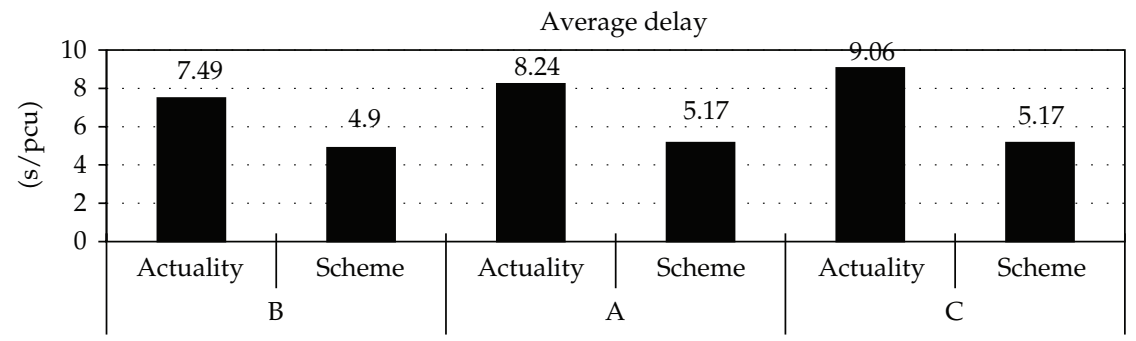

(a)

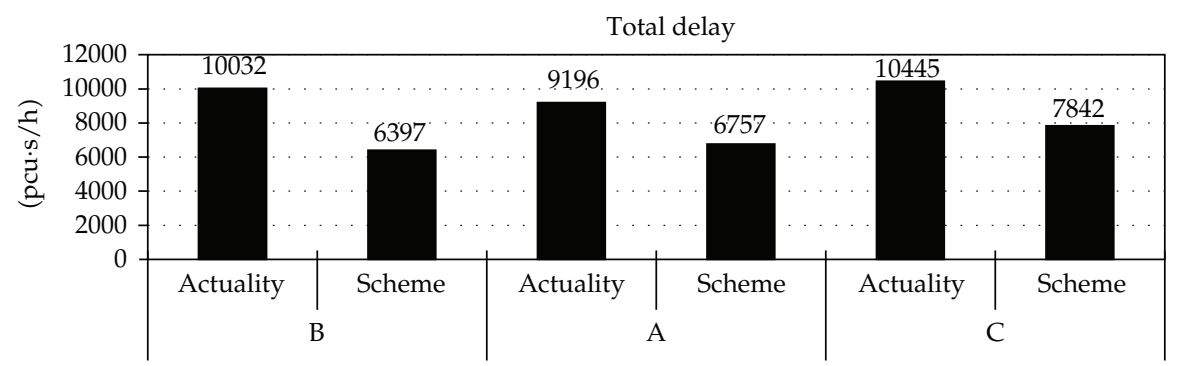

(b)

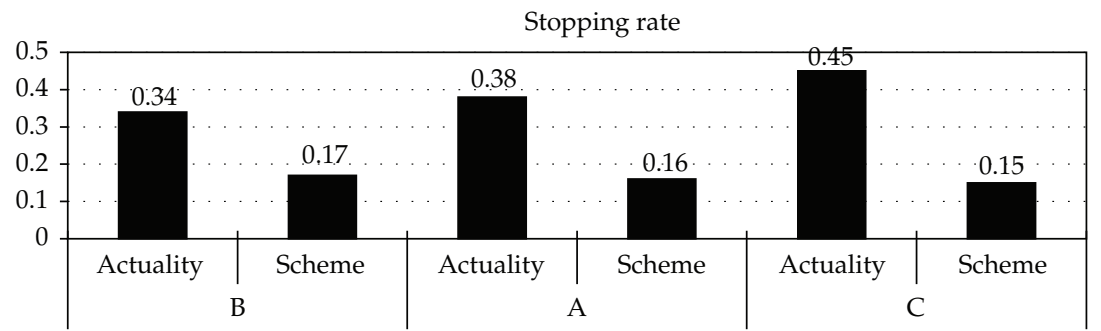

(c)

Figure 11: A comparison figure of vehicle delays from south to north of road actuality and scheme.

C stop line, and the controls parameter optimization model formula (2.6) and (2.7) iteration, we can get that OFS is $35 \mathrm{~s}, C_{p 1}$ is $73 \mathrm{~s}, C_{p 2}$ is $75 \mathrm{~s}$, and $\min D_{r}$ is $24 \mathrm{~s} /$ cycle. Then $t_{c 1}$ is $16 \mathrm{~s}$ and $t_{c 2}$ is $16 \mathrm{~s}$. The $5 \mathrm{~s}$ at the end of two green lights is designed into green flash time.

Through the delay calculating method of actuated signal vehicles and taking saturation flow rate as $0.75 \mathrm{pcu} / \mathrm{s}$, the total vehicle delay $D$ is $322 \mathrm{pcu} \cdot \mathrm{s} / \mathrm{cycle}$, that is $7842 \mathrm{pcu} \cdot \mathrm{s} / \mathrm{h}$. The average delay of vehicles at $C$ is $\bar{D}=D / Q=5.17 \mathrm{~s} / \mathrm{pcu}$ and stopping rate is 0.15 . 


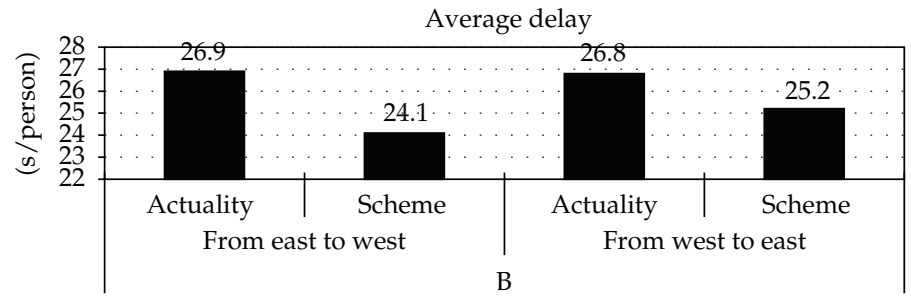

(a)

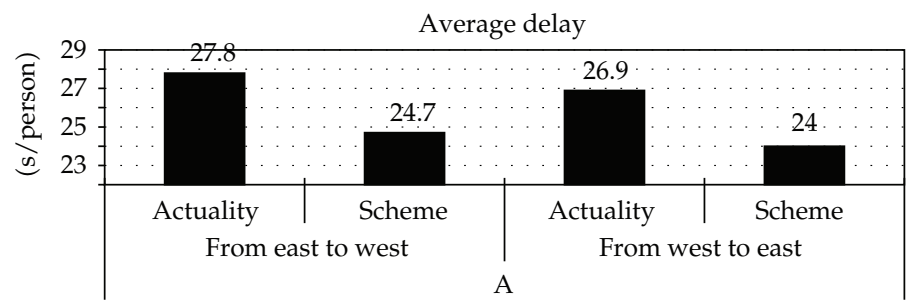

(b)

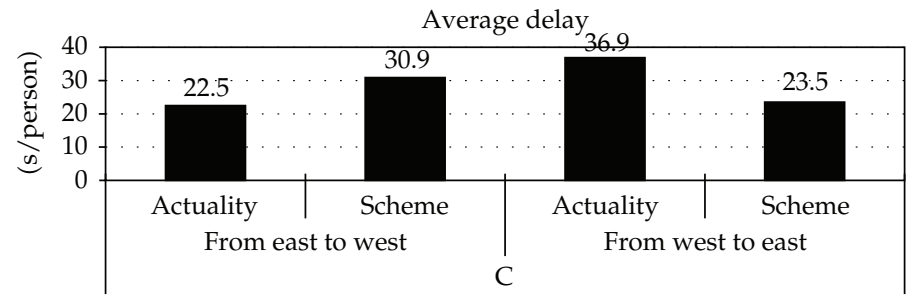

(c)

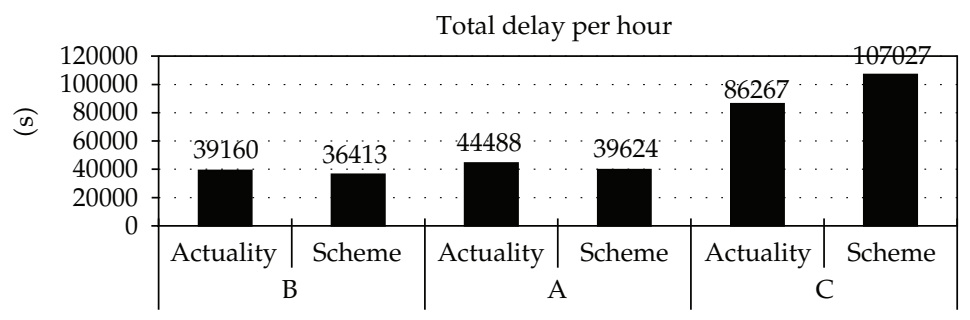

(d)

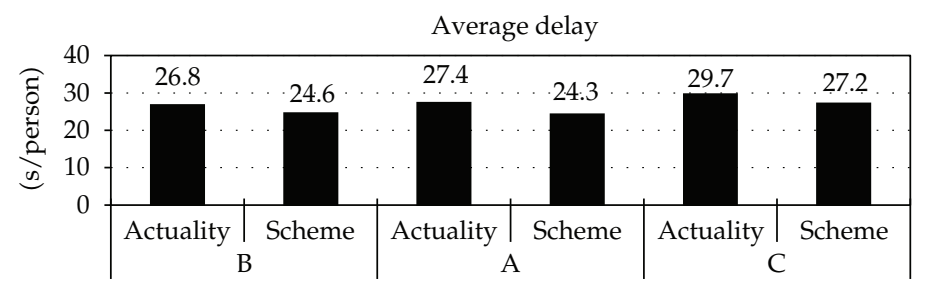

(e)

Figure 12: A comparison figure of pedestrian delays of road actuality and scheme. 
By (3.3) and (3.4), we can get that the average delay is $30.9 \mathrm{~s} /$ person from east to west, $23.5 \mathrm{~s} /$ person from west to east, and the total average delay is $27.2 \mathrm{~s} /$ person. Pedestrian total delay per hour of crosswalk $\mathrm{C}$ is $107027 \mathrm{~s}$.

\subsubsection{Scheme Evaluation}

According to the results of traffic actuality surveys and scheme output, a comparison table of vehicle delays and pedestrian delays from south to north of road actuality and scheme is gained and shown in Figures 11 and 12.

From Figures 11 and 12, it can be seen that vehicles' time benefits evaluation indexes which are vehicle delay and average delay of three crosswalks significantly decrease. And pedestrian mid-block street crossing time benefits evaluation indexes which are the average delay and total delay also decrease obviously after the scheme implemented. Time benefits of the scheme are obviously better than the current situation of signal scheme.

The time-efficient evaluation results of the vehicle from south to north are acquired only considering the vehicle unidirectional (from south to north) delay decreases. And the time-efficient evaluation of vehicles from north to south can also be acquired by the same method.

\section{Conclusion}

To coordinate the signal control between pedestrian mid-block street crossings and intersections, this paper proposes to use "distance-flow rate-time" graph as a tool to establish the system coordination model. The model for calculating delays of vehicles and passengers is established by using the method of coordinating the signal control between pedestrian mid-block street crossings and intersections.

Finally, taking one section on the Taiping North Road in Nanjing as an example, this paper carries out the coordination control of two timing-type crosswalks, a pushbutton (actuated) crosswalk, and the upstream intersection signal of Taiping Road and Zhujiang Road from south to north. Using the most optimal control plan, the system running parameters that are vehicles' delays, stop rate, and passengers' delays are obtained from the model. And though compared to current vehicles' delays at intersections from north to south, we conclude that the coordination control plan this paper proposes is effective.

\section{Acknowledgments}

The authors are grateful to the referee for the comments. This work is supported by the Jiangsu Province Natural Science Foundation (Bk2011745 of China, National key basic research and development program (973 program) issue (2012CB725402) of China).

\section{References}

[1] W. Dian-Hai, Traffic Flow Theory, China Communications Press, Beijing, China, 2002.

[2] M. Hosoi, S. Ishijima, and A. Kojima, "Dynamical model of a pedestrian in a crowd," in Proceedings of the 5th IEEE International Workshop on Robot and Human Communication (RO-MAN), pp. 44-49, November 1996.

[3] W. Wang, W. Zhang, H. Guo, H. Bubb, and K. Ikeuchi, "A safety-based approaching behavioural model with various driving characteristics," Transportation Research Part C, vol. 19, no. 6, pp. 1202 1214, 2011. 
[4] W. H. Wang, Q. Cao, K. Ikeuchi, and H. Bubb, "Reliability and safety analysis methodology for identification of drivers' erroneous actions," International Journal of Automotive Technology, vol. 11, no. 6, pp. 873-881, 2010.

[5] M. S. Tarawneh, "Evaluation of pedestrian speed in Jordan with investigation of some contributing factors," Journal of Safety Research, vol. 32, no. 2, pp. 229-236, 2001.

[6] Y. Pei-Kun and Z. Shu-Sheng, Traffic Managment and Control, China Communications Press, Beijing, China, 2003.

[7] Criterion of Urban Transportation Planning (CJJ37-90), China Construction Industry Press, Beijing, China, 1991.

[8] D. Sun, S. V. S. K. Ukkusuri, and R. F. Benekohal, "Modeling of motorist-pedestrian interaction at uncontrolled mid-block crosswalks," in Proceedings of the TRB Annual Meeting, 2003.

[9] B. Wolshon and W. C. Taylor, "Analysis of intersection delay under real-time adaptive signal control," Transportation Research Part C, vol. 7, no. 1, pp. 53-72, 1999.

[10] M. B. Trabia, M. S. Kaseko, and M. Ande, "A two-stage fuzzy logic controller for traffic signals," Transportation Research Part C, vol. 7, no. 6, pp. 353-367, 1999.

[11] Y. Huang, Y. Kang, and S. Zhao, "Traffic signal coordination control method for river-crossing road bridge under load-bearing restriction," in Proceedings of the International Conference on Transportation, Mechanical, and Electrical Engineering, pp. 601-604, 2011.

[12] L. Canqi and Y. Peikum, "Diffusion models of traffic platoon on signal-intersection and control of coordinated signals," Journal of Tongji University, vol. 24, no. 6, pp. 635-641, 1996.

[13] C. Sen-Fa, C. Hong, and X. Ji-Qian, "Two level fuzzy control and simulation for urban traffic single intersection," Journal of System Simulation, vol. 10, no. 2, pp. 35-40, 1998.

[14] C. Yun-Tao and P. Guo-Xiong, "Urban arterial road coordinate control based on genetic algorithm," Journal of Traffic and Transportation Engineering, vol. 3, no. 2, pp. 106-112, 2003.

[15] X. Chu, M. Guttenplan, and M. Baltes, "Why people cross where they do:the role of the street environment," in Proceedings of the TRB Annual Meeting, 2003.

[16] J. Ye, X. Chen, and N. Jian, "Impact analysis of human factors on pedestrian traffic characteristics," Fire Safety Journal, vol. 52, pp. 46-54, 2012.

[17] Q. Yong-shen, Urban Traffic Control, China Communications Press, Beijing, China, 1989.

[18] Y. Xiao-Guang, C. Bai-Lei, and P. Guo-Xiong, "Study of the way of setting pedestrian's traffic control signal," China Journal of Highway and Transport, vol. 14, no. 1, pp. 73-80, 2001.

[19] W. Wei and G. Xiu-Cheng, Traffic Engineering, Southeast University Press, Nanjing, China, 2000.

[20] Z. Zu-Wu, Modern Traffic in City, China Communications Press, Beijing, China, 1998. 


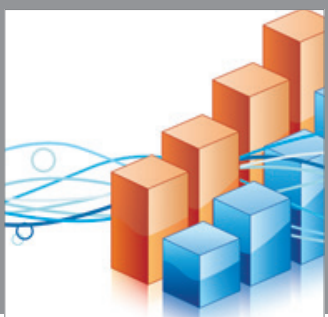

Advances in

Operations Research

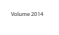

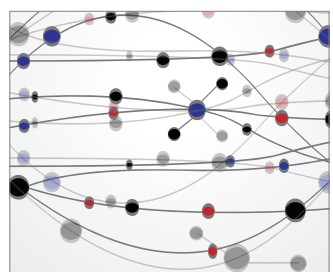

\section{The Scientific} World Journal
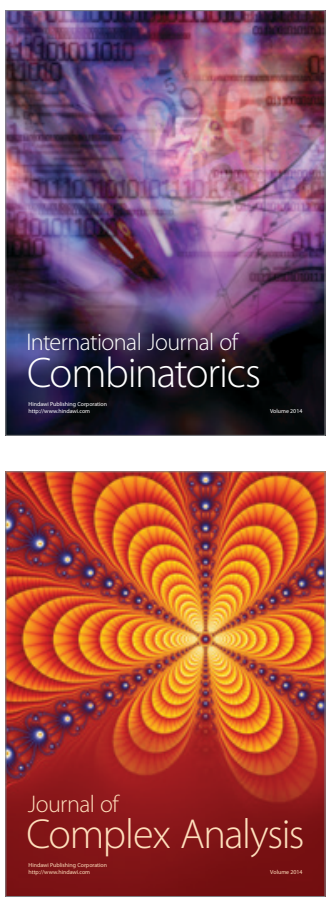

International Journal of

Mathematics and

Mathematical

Sciences
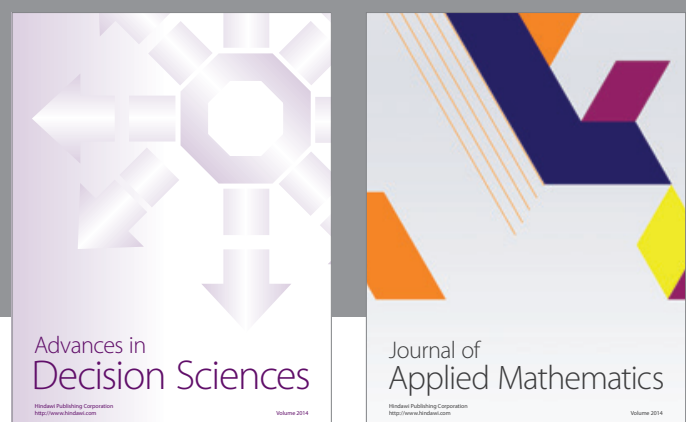

Journal of

Applied Mathematics
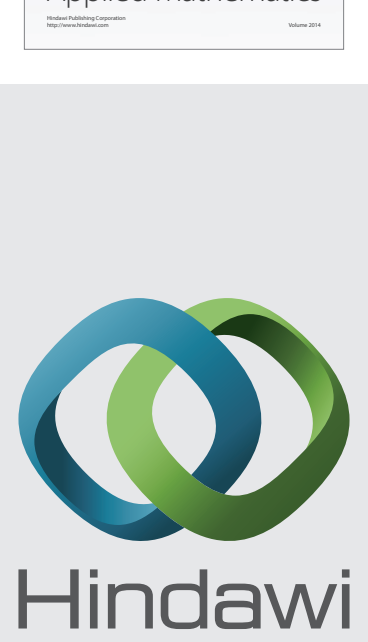

Submit your manuscripts at http://www.hindawi.com
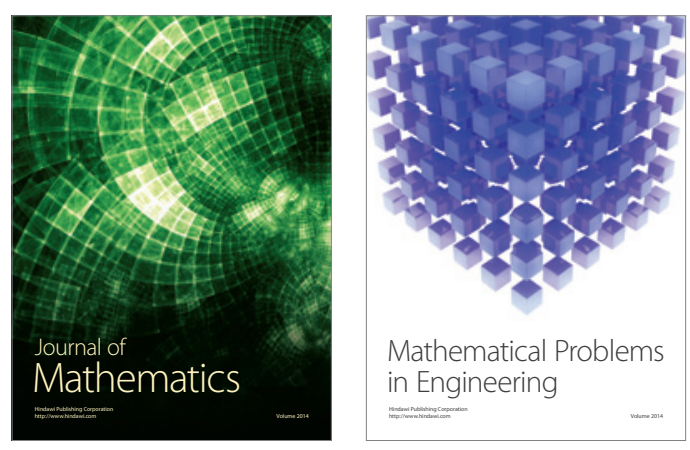

Mathematical Problems in Engineering
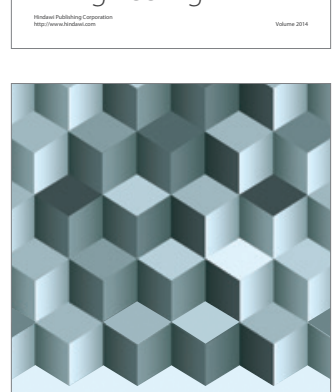

Journal of

Function Spaces
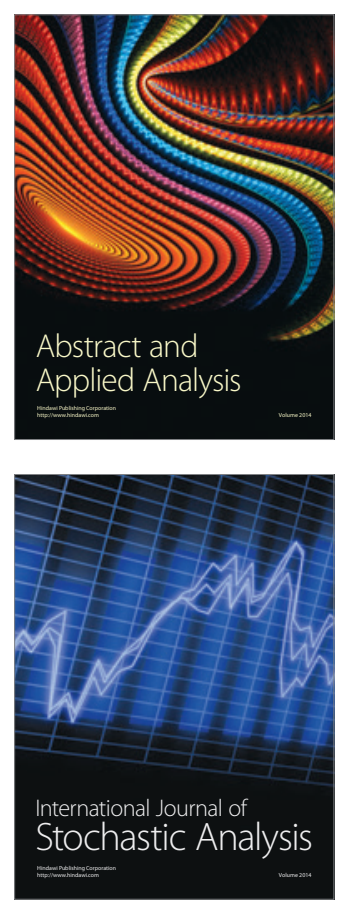

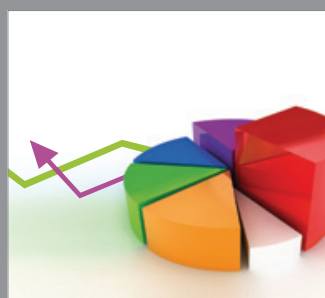

ournal of

Probability and Statistics

Promensencen
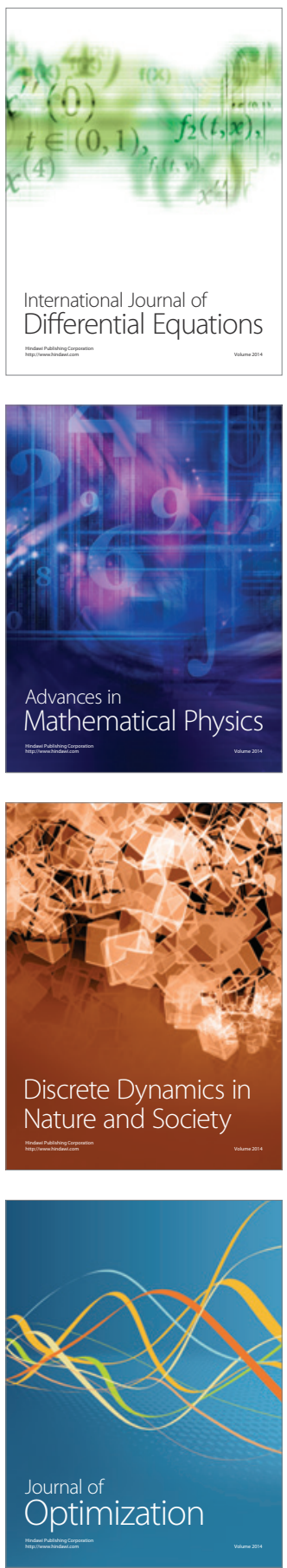